\title{
Alveolar Bone Changes in Post-menopausal Osteopenic and Osteoporosis Women: An Original Research
}

\author{
Majumder M I', Harun M A S I²
}

\author{
${ }^{1}$ Department of Medicine, Comilla Medical College, Comilla, Bangladesh, ${ }^{2}$ Department of Conservative Dentistry \\ and Dental Radiology, Chattagram International Dental College, Chattagram, Bangladesh
}

\begin{abstract}
Background: Osteoporosis is imposing public health burden especially in postmenopausal women and elderly population. It is leading cause of morbidity and mortality in postmenopausal women. Among multiple modalities of osteoporosis diagnosis, bone mineral density (BMD) is considered as a gold standard by WHO. Aim: Evaluate the alveolar bone changes in osteopenic and osteoporosis condition of postmenopausal women. Materials and Methods: Totally 1315 postmenopausal women were included in this study. All patients were evaluated by dual-energy X-ray absorptiometry for BMD and dental radiograph such as orthopantomography and radiovesiography. Among those women 72 were normal, 276 were osteopenic and 967 were osteoporotic. Mandibular cortical index (MCl), pixel intensity (PI), mandibular alveolar bone mass (MABM), and Alveolar bone resorption pattern (ABRP) were evaluated from the dental radiograph. Calculation of mean and standard deviation, as well as correlation and difference, were performed using SPSS 11.5 for Windows (Windows XP). Results: The changes in the $\mathrm{MCl}(\mathrm{C} 2$ and $\mathrm{C} 3$ ) were more in osteoporotic condition $(87.48 \%)$ rather than osteopenic $(31.34 \%)$ and normal $(12.50 \%)$. The numbers of presenting teeth were more in normal and osteopenic women rather than osteoporotic women. $\mathrm{PI}$ and MABM were significantly different from normal vs. osteopenic and osteoporosis, osteopenic versus osteoporosis. Horizontal ABRP was more in osteoporosis women (91.21\%). Conclusion: Changes in postmenopausal alveolar bone were strongly correlated with the BMD of systemic skeletal bone. This combined relationship can be used as an easy diagnosis tool for innovations in osteoporotic condition.
\end{abstract}

Key words: Bone mineral density, dental radiology, duel X-ray absorptiometry, osteopenia, osteoporosis, postmenopause

\section{INTRODUCTION}

Osteoporosis is a medical disorder characterized by a generalized low bone mess and fragility with a consequent increase in fracture risk, ${ }^{[1]}$ particularly of vertebrae, hip, and wrist. ${ }^{[2]}$ It is a physiological

\begin{tabular}{|c|c|}
\hline \multicolumn{2}{|c|}{ Access this article online } \\
\hline Publisher & \multirow[b]{2}{*}{$\begin{array}{l}\text { Website: } \\
\text { www.renupublishers.com }\end{array}$} \\
\hline & \\
\hline & $\begin{array}{l}\text { DOI: } \\
\text { 10.5958/2394-4196.2015.00010.2 }\end{array}$ \\
\hline
\end{tabular}

gender and age-related condition resulting from bone mineral content loss and structural changes in bones. There are two types of osteoporosis: (1) Postmenopausal osteoporosis caused by cessation of estrogen production and characterized by spinal fracture and (2) Osteoporosis that affect an older population and results in proximal femur fracture. ${ }^{[3]}$

In future projection, the World population is expected to rise from the current 323 million individuals aged 65 years to more than 1.5 billion by the year 2050 . These demographic changes alone can be expected to increase the number of hip fractures occurring worldwide. This incidence is estimated to rise from 1.66 million in 1990

\footnotetext{
Address for Correspondence:

Harun M A S I, Department of Conservative Dentistry and Dental Radiology, Chattagram International Dental College, Shamsher para, Chandgaue, Chattagram, Bangladesh. Phone: +91-880171115586. E-mail: drharunpg@yahoo.com
}

Submission: 05 Mar 2015; Revision: 20 Apr 2015; Acceptance: 13 May 2015 
to 6.26 million in 2050. ${ }^{[4]}$ A conservative estimate of the worldwide direct and indirect annual cost of hip fracture for the year 1990 was 34.8 billion US dollar. This is set to rise to 131.5 billion in US dollars by 2050 (at a cost of 21000 per patient). ${ }^{[4,5]}$

Osteoporosis diagnosis and staging are based on the identification of different risk factors, the most important being low bone mineral density (BMD) of the femoral neck or lumber spine. ${ }^{[6]}$ WHO has been established four diagnostic levels of BMD: (1) The normal bone when $t$ score is better than -1 $\left(>833 \mathrm{mg} / \mathrm{cm}^{2}\right)$. (2) Osteopenia when $t$ score are between $\geq 1$ and 2.5 (between 833 and $648 \mathrm{mg} / \mathrm{cm}^{2}$ ). (3) Osteoporosis when $t$ score is $<-2.5$ (lower than $648 \mathrm{mg} / \mathrm{cm}^{2}$ ). (4) Established osteoporosis when nontraumatic fractures are includes and $t$ score is $<-2.5$. $^{[7]}$

Suggestion have been made that panoramic radiograph that show progressive periodontal disease, alveolar bone, tooth loss and endosteal resorption of the mandibular inferior cortex may indicate general osteoporosis. ${ }^{[5]}$ The uses of panoramic radiographs are common a dental sitting. Digital radiographs are an increasingly popular option in the clinic. Such images are composed of the pixel with a specific numerical value for each one. Two important methods of evaluating the pixel in these images are a fractal dimension and pixel intensity (PI) ${ }^{[8]}$

PI is a grayscale measure, ranging from 0 (black) to 256 (white) in a digital image. Areas of bone lose represented as darker areas while areas of the bone gain represented as lighter area. ${ }^{[9]}$

The alveolar bone is a unique tissue representing the mast viable part of the tooth-supporting apparatus. The alveolar process consists of an external plate of cortical bone, the inner socket of thick compact bone, and cancellous trabeculae interposed. Alveolar bone is intramembranous in origin and undergoes continuous remodeling by osteoblast and osteoclast activity. ${ }^{[9,10]}$

Young alveolar bone is dense bone with smooth walled socket, while aged alveolar bone is osteoporotic with a rough, jagged socket wall; fewer viable cells in lacunae, marrow tissue infiltrated by fat cells and thus diminished tooth support. ${ }^{[1]}$

Tooth function is a prerequisite for the maintenance of the alveolar bone and cementum. Loss of alveolar bone (metabolic disease/osteoporosis/aging/periods of inactivity) is always accompanied by loss of periodontal fibers. Periodontal disease is among one of the oral problems that most extensively affect the human population, being one of the major cause for adult tooth loss. ${ }^{[12]}$

Alveolar bone exists to support the teeth. Its structure varies between individual and generally it gets denser with age ${ }^{[11]}$ Broadly, there is a dense bone wall near the gingivae and then middle spaces near the tooth apex. ${ }^{[12]}$

Osteoporosis may arise in the context of other disease such as inflammatory bowel disease or primary biliary cirrhosis, as the result of medication, most commonly steroids or as a consequence of postmenopausal aging. ${ }^{[13-15]}$

Many studies have cited the possible correlation between age, systemic osteoporosis, periodontal disease, tooth loss and changes in quantity and quality of bone of the maxillae and mandible. The restoration of occlusion for partially and the totally edentulous patient often requires adequate bone therapy. Consequently, the frequent use of implant supported prosthesis for elderly patient who are routinely or potentially osteoporotic demand a better understanding of the relationship between osteoporosis, the stomatognatic system. The purpose of this study was evaluating the alveolar bone changes in osteopenic and osteoporosis condition of postmenopausal women.

\section{MATERIALS AND METHODS}

Totally 1315 postmenopausal women were included in this study with the complained of osteoporotic symptoms. Patients with suspected conditions affecting bone mineralization or receiving any treatment affecting bone mineralization were excluded. All patients were evaluated by dual energy X-ray absorptiometry (DEXA) for BMD, and dental radiograph such as orthopantomography (OPG), radiovesiography (RVG). Among 1315 postmenopausal women 967 were osteoporotic, 276 osteopenic and 72 normal in condition.

Osteoporosis and Osteopenic were diagnosis by DEXA scans from Comilla Medical College, Bangladesh. BMD at lumber spine and femoral neck were measured by DEXA scanner.

All Panoramic radiographs are obtained at the time of the DEXA scan from Central Medical College and 
Hospital, Comilla. Some visual points were seen in panoramic radiograph there are as follows.

\section{Direction of Bone Resorption}

If bone loss is suggested on the films, it may be "horizontal" or "vertical" in character. Horizontal bone loss is indicated when the bone loss interproximally on two adjacent tooth, vertical bone loss is indicated when the bone crest is more apical to the cementoenamel junction adjacent to one tooth than to other.

\section{Mandibular Cortical Index (MCI)}

Panoramic radiograph has been analyzed for MCI. According to Klemitti et al. MCI is classification of appearance of the mandibular inferior cortex distal to the mental foramen, which includes the following criteria. ${ }^{16}$

- $\mathrm{C}_{1}$ : The endosteal margin of the cortex is even sharp on both sides of the mandible.

- $\mathrm{C}_{2}$ : The endosteal margin has semilunar defects (resorptive cavities) with cortical residues one to three layers thick on one or both sides.

- $\mathrm{C}_{3}$ : The endosteal margin consists of thick cortical residues and is clearly porous.

\section{Digital Radiograph}

After the clinical examination, periapical radiograph were taken by using RVG. RVG are manufactured by Gendex, USA. Vixwin Platinum image processing software is used for the measurement of gray level of bone. Digital periapical radiograph by RVG was used due to their minimal radioactive emission and high image quality that are not lost upon digitalization.

\section{Mandibular Alveolar Bone Mass (MABM)}

The MABM by the mean gray level values of the alveolar bone on the digital radiograph. The region of interests (ROIs) were set on the apical radiograph of the individual on the $6 \mathrm{~mm}$ step of the reference radiograph with "rectangular tool" avoiding the lamina dura and the most crestal locations. No apical bone was included. ${ }^{[6]}$ Then the ,PI were measured from low level to high level (by assigning the value 0 to black 256 to white). Areas of bone loss represent as darker while areas of the bone gain represented as lighter areas. ${ }^{[6]}$ MABM were evaluated from the mean value of PI. This obtained data are presented as mean \pm standard deviation (SD) and it was posted to the data sheet for statistical analysis.
Calculation of mean and SD, as well as correlation and difference, were performed using SPSS 11.5 for windows (Windows XP). $P<0.05$ were considered to be statistically significant.

\section{RESULT}

The result showed that the BMD of lumbar spine and femoral neck were $-0.76 \pm 0.38$ and $-0.49 \pm 0.34$ respectively with PI level $81.40 \pm 26.90$ to $128.65 \pm 31.92$, MABM were $104.5 \pm 14$, horizontal alveolar bone resorption (HABR) were $48.61 \%$, and mandibular cortical changes (MCI - C2 and C3) were $12.5 \%$ in normal postmenopausal patients. Whereas in osteopenic patients the BMD of lumbar spine and femoral neck were $-1.88 \pm 0.6$ and $-1.25 \pm 0.73$ respectively with PI level $70.02 \pm 29.22$ to $1113.25 \pm 33.62$, MABM were $91.5 \pm 12.84$, HABR were $72.10 \%$, and mandibular cortical changes (MCI - C2 and C3) were 31.34\%. But in osteoporosis patients the BMD of lumbar spine and femoral neck were $-3.47 \pm 0.99$ and $-2.73 \pm 1.65$ respectively with PI level $63.63 \pm 28.37$ to $104.73 \pm$ 32.86, MABM were $83.5 \pm 12.27$, HABR were $91.21 \%$, and mandibular cortical changes (MCI - C2 and C3) were $87.48 \%$. In statistical analysis $P<0.05$. All data showed BMD and dental radiographic finding were running parallel [Table 1].

\section{DISCUSSION}

In this study, we found that mean BMD in three types of condition were significantly different from each others. Other associated variables like age, weight and height were also significantly different $(P<0.05)$ except age when compare between normal and osteopenic patient $(P=0.24)$. Alterations in the mandibular alveolar bone (bone mass, PI, presenting teeth, alveolar bone resorption pattern $[\mathrm{ABRP}]$ ) were related to the $\mathrm{BMD}$ level. The golden standard for fracture risk is BMD of the proximal femur measured with DXA. ${ }^{[16]}$ This study was to compare alveolar bone changes with skeletal bone change in postmenopausal patient.

\section{PI changes}

The PI of alveolar bone in normal postmenopausal patients was significantly different from osteopenic and osteoporosis patients. The minimum and maximum level of PI were reduced in both osteopenic and osteoporosis patients when compare to the normal postmenopausal condition [Tables 2 and 3]. In osteoporosis patients, 
the minimum and maximum level of PI were reduced significantly from osteopenic patients [Table 4].

The deficit in bone formation in cortical bone result in increased number of lacunae and porosities and later on in thinner cortical plates ${ }^{[17-19]}$ it leads to larger intertrabecular spaces and thinning of the trabeculae in the cancellous bone. ${ }^{[20]}$ When the cortices and trabeculae are thinner, and intertrabecular spaces are larger in a certain area (ROI), the mineral content is decreased, and therefore, also the PI was decreased.

\section{Mandibular Alveolar Bone Mass}

The mandibular alveolar mass of normal postmenopausal patient were significantly different from osteopenic and osteoporosis patients. MABM was reduced significantly reduced with the level of femoral and lumbar BMD. Although the MABM were calculated from PI, it is not mandatory to changes with PI. Because the changes in BMD were associated with changes in the PI of the mandibular alveolar bone measured between premolars, but not with changes in MABM. ${ }^{[9]}$ In other study showed the mandibular bone mass were improved with the parallel improvement of BMD after treatment with zoledronic acid. ${ }^{[21]}$

\section{Presenting Teeth}

The number of presenting teeth in postmenopausal normal and osteopenic patients were not significantly difference. But in postmenopausal osteoporotic patients, the number of presenting teeth were significantly reduced when compare to the normal and osteopenic patients.

In another study, association between dental status and skeletal bone density were investigated in a group of 329 healthy postmenopausal women with normal bone density. Significant positive linear relationships were observed between number of teeth and BMD at the spine and radius. ${ }^{[22]}$ Some others study had establish a significant association between osteoporosis and tooth loss, ${ }^{[23]}$ mandibular bone mass, and tooth loss. ${ }^{[19]}$

\section{Mandibular Cortical Index}

Several radiographic indices in the mandible on OPG have been developed to assess general skeletal BMD or osteoporosis status: MCI, PMI, gonial index (GI), antegonial index (AI), mental index (MI) and the ratio of alveolar bone height to basal bone height of the mandible. ${ }^{[24-27]}$ The general dental practitioner will

Table 1: Age, weight, height, lumber and femoral BMD

\begin{tabular}{|c|c|c|c|c|c|}
\hline \multirow[t]{2}{*}{ Variables } & \multirow[t]{2}{*}{ Age } & \multirow[t]{2}{*}{ Weight } & \multirow[t]{2}{*}{ Height } & Lumber BMD & Femoral BMD \\
\hline & & & & $t$ score $\left(\mathrm{g} / \mathrm{cm}^{2}\right)$ & $t$ score $\left(\mathrm{g} / \mathrm{cm}^{2}\right)$ \\
\hline Normal & $54.47 \pm 6.55$ & $60.63 \pm 9.52$ & $152.75 \pm 7.01$ & $-0.76 \pm 0.38(1.10 \pm 0.10)$ & $-0.49 \pm 0.34(1.016 \pm 0.08)$ \\
\hline Osteopenia & $55.75 \pm 8.8$ & $55.47 \pm 9.98$ & $149.27 \pm 5.81$ & $-1.88 \pm 0.6(0.924 \pm 0.11)$ & $-1.25 \pm 0.73(0.887 \pm 0.12)$ \\
\hline Osteoporosis & $62.06 \pm 10.45$ & $47.52 \pm 9.3$ & $147 \pm 7.46$ & $-3.47 \pm 0.99(0.705 \pm 0.13)$ & $-2.73 \pm 1.65(0.668 \pm 0.13)$ \\
\hline
\end{tabular}

BMD: Bone mineral density

Table 2: Changes in normal postmenopausal patients versus osteopenic patients

\begin{tabular}{|c|c|c|c|c|c|c|}
\hline Variables & Presenting teeth & PI (min) & $\mathrm{PI}(\max )$ & MABM & $\mathrm{MCl}(\mathrm{C} 2$ and C3) $n(\%)$ & HABR $n(\%)$ \\
\hline Normal & $28.37 \pm 4.52$ & $81.40 \pm 26.90$ & $128.65 \pm 31.92$ & $104.5 \pm 14$ & $9(12.50)$ & $35(48.61)$ \\
\hline Osteopenic & $28.18 \pm 7.77$ & $70.02 \pm 29.22$ & $113.25 \pm 33.92$ & $91.5 \pm 12.84$ & $131(31.34)$ & $199(72.10)$ \\
\hline$P<0.05$ & 0.842 & 0.003 & 0.0005 & 0.0001 & & \\
\hline
\end{tabular}

PI: Pixel intensity, MABM: Mandibular alveolar bone mass, MCI: Mandibular cortical index, HABR: Horizontal alveolar bone resorption

Table 3: Changes in normal postmenopausal patients versus osteoporosis patients

\begin{tabular}{lcccccc}
\hline Variables & Presenting teeth & PI $(\mathbf{m i n})$ & PI $(\max )$ & MABM & MCI $(\mathbf{C} 2$ and C3) $\boldsymbol{n}(\%)$ & HABR $\boldsymbol{n}(\%)$ \\
\hline Normal & $28.37 \pm 4.52$ & $81.40 \pm 26.90$ & $128.65 \pm 31.92$ & $104.5 \pm 14$ & $9(12.50)$ & $35(48.61)$ \\
Osteoporosis & $24.91 \pm 8.25$ & $63.63 \pm 28.37$ & $104.73 \pm 32.86$ & $83.5 \pm 12.27$ & $846(87.48)$ & $882(91.21)$ \\
$P<0.05$ & 0.0005 & 0.0001 & 0.0001 & 0.0001 & & \\
\hline
\end{tabular}

PI: Pixel intensity, MABM: Mandibular alveolar bone mass, MCl: Mandibular cortical index, HABR: Horizontal alveolar bone resorption

Table 4: Changes in osteoporosis patients versus osteopenic patients

\begin{tabular}{|c|c|c|c|c|c|c|}
\hline Variables & Presenting teeth & $\mathrm{PI}$ (min) & $\mathrm{PI}$ (max) & MABM & $\mathrm{MCl}(\mathrm{C} 2$ and $\mathrm{C} 3) n(\%)$ & HABR $n(\%)$ \\
\hline Osteopenic & $28.18 \pm 7.77$ & $70.02 \pm 29.22$ & $113.25 \pm 33.92$ & $91.5 \pm 12.84$ & $131(31.34)$ & 199 (72.10) \\
\hline Osteoporosis & $24.91 \pm 8.25$ & $63.63 \pm 28.37$ & $104.73 \pm 32.86$ & $83.5 \pm 12.27$ & $846(87.48)$ & $882(91.21$ \\
\hline$P<0.05$ & 0.0001 & 0.0001 & 0.0001 & 0.0001 & & \\
\hline
\end{tabular}

PI: Pixel intensity, MABM: Mandibular alveolar bone mass, MCl: Mandibular cortical index, HABR: Horizontal alveolar bone resorption 
tend to select women with $\mathrm{C} 3$ cortex rather than $\mathrm{Cl}$ and $\mathrm{C} 2$ cortex on OPG for identifying women with low BMD. ${ }^{[28]}$

In our study, the mandibular cortex (C2 and C3) were more changes in osteoporotic patients rather than osteopenic patients. On the other hand, few numbers of normal postmenopausal patients had showed the mandibular cortical changes (C2 and C3). Determine the diagnostic validity of the MCI for the diagnosis of osteoporosis in peri and post-menopausal women, MCI had low sensitivity but high specificity for the diagnosis of osteoporosis. ${ }^{[29]}$

Although some study could not find any correlation with the skeletal BMD, ${ }^{[30]} \mathrm{MCI}$ is relatively simple because no measurements of calculation are required but it depends on visual assessment. Its repeatability has been evaluated and especially inter-observer agreement is reported to be poor. ${ }^{[30,31]}$ For this reason, single observer for MCI evaluation was used in this study.

\section{Alveolar Bone Resorption Pattern}

Horizontal bone resorption is the most common pattern of bone loss in periodontal disease. ${ }^{[32]}$ The present study showed the number of horizontal bone resorption pattern were increased in postmenopausal osteoporotic patients rather than osteopenic and normal patients.

The systemic influence on the response of alveolar bone has been termed as the "bone factor concept" in periodontal diseases. ${ }^{[33]}$ In recent years, lot of studies focused on the possible relationship between periodontal bone loss and osteoporosis. ${ }^{[34]}$

\section{CONCLUSION}

Changes in the alveolar bone mass in postmenopausal women's were strongly correlated in this study with the BMD level of skeletal bone. Though BMD is the gold standard for diagnosis and follow-up of osteoporosis, Dental radiographical finding comprising of PI, MCI, MABM, and ABRP determination can be used as diagnostic tool in post-menopausal osteoporosis and osteopenic women and for follow-up. Hence, it was concluded that this combined relationship can be utilized as a logarithm for innovations in making a smooth diagnostic tools in post-menopausal osteoporotic women.

\section{REFERENCES}

1. Ismal NM. Postmenopausal osteoporosis: Epidemiology, pathophysiology and treatment. Malays J Pathol 1997;19:21-5.

2. Rizzoli R, Bruyere O, Cannata-Andia JB, Lyritis G, Ringe JD, Vellas B, et al. Managment of osteoporosis in the elderly. Curr Med Res Opin 2009;25:2373-87.

3. Riggs BL, Melton LJ. Involution osteoporosis. N Engl J Med 1986;26;1676-86.

4. Sambrook Philip N. Incidence of Hip Fracture set to Increase to 6.3 Million Worldwide in 2050. Eurek Alert. June 16,2006. Available from: http://www.eurekalert.org/pub_releases/2006-06/1-ioh061406. php.00:01H (London time). [Last accessed on 2006 Jun 15].

5. Cooper C, Melton LJ. Magnitude and impact of osteoporosis and fracture. In: Marcuse R, Feldman D, Kelsey J, editors. Osteoporosis. San Diago, CA: Academic Press; 1996. p. 419-34.

6. Tosoni GM, Lurie AG, Cowan AE, Burleson JA. Pixel intensity and fractal analyses: Detecting osteoporosis in perimenopausal and postmenopausal women by using digital panoramic images. Oral Surg Oral Med Oral Pathol Oral Radiol Endod 2006;102:235-41.

7. World Health Organization. Diet, Nutrition, and the Prevention of Chronic Diseases. Geneva: World Health Organization; 2003. (WHO Technical Report Series, 916).

8. Von Muhan D, Visby L, Barret-Connor E, Bettencourt R. Evaluation of the sample calculated osteoporosis risk estimation (SCORE) in order Caucasian woman: The Rancho Bernardo study. Osteoporosis Int 1999;10:79-83.

9. Jonasson G, Bankvall G, Kiliaridis S. Estimation of skeletal bone mineral density by means of the trabecular pattern of the alveolar bone, its interdental thickness, and the bone mass of the mandible. Oral Surg Oral Med Oral Pathol Oral Radiol Endod 2001;92:346-52.

10. Kanis JA. Assessment of fracture risk and its application to screening for postmenopausal osteoporosis: Synopsis of a WHO report. WHO Study Group. Osteoporos Int 1994;4:368-81.

11. von Wowern N, Klausen B, Kollerup G. Osteoporosis: A risk factor in periodontal disease. J Periodontol 1994;65:1134-8.

12. Marcelo RM, da Silva MA, Barros SP. Periodontal disease and osteoporosis associated and mechanism; A review of literature. Braz J Oral Sci 2003;2:20-5.

13. Zein CO, Jorgensen RA, Clarke B, Wenger DE, Keach JC, Angulo P, et al. Alendronate improves bone mineral density in primary biliary cirrhosis: A randomized placebo-controlled trial. Hepatology 2005;42:762-71.

14. Jonasson G, Jonasson L, Kiliaridis S. Changes in the radiographic characteristics of the mandibular alveolar process in dentate women with varying bone mineral density: A 5-year prospective study. Bone 2006;38:714-21.

15. Bone HG, Hosking D, Devogelaer JP, Tucci JR, Emkey RD, Tonino RP, et al. Ten years experience with alendronate for osteoporosis in postmenopausal woman. N Engl J Med 2004;350:1189-95.

16. Kanis JA, Johnell O, Oden A, De Laet C, Mellstrom D. Diagnosis of osteoporosis and fracture threshold in men. Calcif Tissue Int 2001;69:218-21.

17. Kribbs PJ, Chesnut CH, Ott SM, Kilcoyne RF. Relationships between mandibular and skeletal bone in an osteoporotic population. J Prosthet Dent 1989;62:703-7.

18. Klemetti E, Kolmako S, Kroger H. Pan tomography is assessment of osteoporosis risk group. Scand Dent Res 1994;102:68-74.

19. Taguchi A, Tanimoto K, Suei Y, Otani K, Wada T. Oral signs as indicators of possible osteoporosis in elderly woman. Oral Surg Oral Med Oral Path Oral Radio Endod 1995;80:612-8.

20. Weinstein RS, Hutson MS. Decreased trabecular width and increased trabecular spacing contribute to bone loss with aging. Bone 1987;8:137-42.

21. Mazumder MI, Harun MA, Shoma AK, Uddin M. Improvement 
of mandibular bone mass in postmenopausal osteoporotic patients with the treatment of zoledronic acid. Int J Dent Med Spec 2014;1:6-9.

22. Krall EA, Dawson-Hughes B, Papas A, Garcia RI. Tooth loss and skeletal bone density in healthy postmenopausal women. Osteoporos Int 1994;4:104-9.

23. Nicopoulou-Karayianni K, Tzoutzoukos P, Mitsea A, Karayiannis A, Tsiklakis K, Jacobs R, et al. Tooth loss and osteoporosis: The osteodent study. J Clin Periodontol 2009;36:190-7.

24. Devlin H, Horner K. Mandibular radiomorphometric indices in the diagnosis of reduced skeletal bone mineral density. Osteoporos Int 2002;13:373-8.

25. Wical KE, Swoope CC. Studies of residual ridge resorption. Part I. Use panoramic radiographs for evaluation and classification of mandibular resorption. J Prosthet Dent 1974;32:7-12.

26. Bras J, van Ooij CP, Abraham-Inpijn L, Wilmink JM, Kusen GJ. Radiographic interpretation of the mandibular angular cortex: A diagnostic tool in metabolic bone loss. Part II. Renal osteodystrophy. Oral Surg Oral Med Oral Pathol 1982;53:647-50.

27. Benson BW, Prihoda TJ, Glass BJ. Variation in adult cortical bone mass as measured by panoramic mandibular index. Oral Surg Oral Med Oral Pathol 1991;71:349-56.

28. Nakamoto T, Taguchi A, Ohtsuka M, Suei M, Fujita M, Tanimoto K, et al. Dental panoramic radiograph as a tool to detect postmenopausal women with low bone mineral density: Untrained general dental practitioner's diagnostic performance. Osteoporos Int 2003;14:659-64.

29. Christina L, Karayianni K, Hornar K, Nackaerts O, Jacobs R, Zhao F, et al. Mandibular cortical index for osteoporosis diagnosis: The Osteodent Project. $10^{\text {th }}$ European Congress of Dentomaxillofacial Radiology, Leuven, Belgium 2006.

30. Horner K, Devlin H. The relationships between two indices of mandibular bone quality and bone mineral density measured by dual energy X-ray absorptiometry. Dentomaxillofac Radiol 1998;27:17-21.

31. Peitgen HO, Jurgens H, Saupe D. Chaos and Fractals. New York: Springer-Rerlag; 1992.

32. Papapanou PN, Tonetti MS. Diagnosis and epidemiology of periodontal osseous lesion. Periodontol 2000;22:8-14.

33. Manson JD. Bone morphology and bone loss in periodontal disease. J Clin Periodontol 1976;3:14-22.

34. Jaftcoat MT, Lewis CE, Reddy MS, Wang CY, Redford M. Postmenopausal bone loss and it's relationship or oral bone loss. Periodontal 2000;23:94-8.

How to cite this article: Majumder MI, Harun MA. Alveolar Bone Changes in Post-menopausal Osteopenic and Osteoporosis Women: An Original Research. Int J Dent Med Spec 2015;2(2):9-14.

Source of Support: Nil; Conflict of Interest: None 